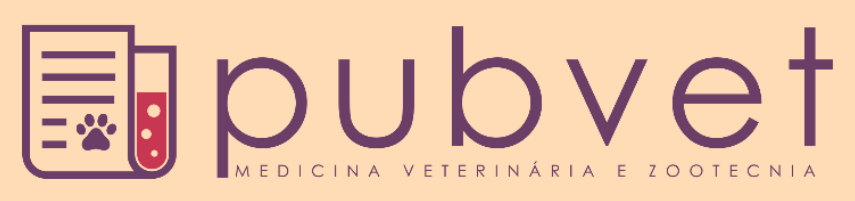

https://doi.org/10.31533/pubvet.v14n2a506.1-5

\title{
Perfil epidemiológico de cães e' gatos intoxicados por rodenticidas em clínica na cidade de Belém, Pará
}

\author{
Hugo Augusto Mendonça Canelas ${ }^{1 * \bullet}$, Amanda Melo Hamoy ${ }^{1}$, Lilian Beatriz Rocha Inajosa $^{1 \bullet}$, \\ Isabela Cristina Santos da Silva ${ }^{\circ}$, Alessandra Souza Negrão ${ }^{2}{ }^{\circ}$, Erica Flávia Silva Azevedo ${ }^{30}$, \\ Dulcideia da Conceição Palheta ${ }^{\circ}$, Paulo Sérgio dos Santos Souto ${ }^{\circ}{ }^{\circ}$, Leony Soares Marinho ${ }^{4}{ }^{\circ}$ \\ ${ }^{I}$ Acadêmico de medicina veterinária na Universidade Federal Rural da Amazônia, Belém-Pa, Brasil. \\ ${ }^{2}$ Médica veterinária autônoma, Belém-Pará, Brasil. \\ ${ }^{3}$ Médica veterinária residente na Universidade Federal Rural da Amazônia, Belém-PA, Brasil \\ ${ }^{4}$ Docentes do curso de medicina veterinária na Universidade Federal Rural da Amazônia, Belém-PA, Brasil \\ *Autor para correspondência, E-mail: canelashugo098@gmail.com
}

\begin{abstract}
Resumo. A principal função do rodenticida é exterminar o respectivo animal, que é um dos principais disseminadores de várias doenças no ambiente urbano importantes para a saúde pública, como a leptospirose. No entanto, na maioria das vezes, essa substância acaba intoxicando animais de estimação e podem ser causadas acidentalmente, quando o tutor não realiza o manejo correto do veneno de rato, ou intencionalmente, quando se destina a causar envenenamento do animal, causando várias sintomatologias em que o prognóstico não é favorável. Devido à alta variedade de sintomas, sua gravidade e alta incidência de casos de intoxicação, foi realizado um levantamento epidemiológico da casuística em uma clínica particular na cidade de Belém/ PA.
\end{abstract}

Palavras chave: animais domésticos, estudo epidemiológico, veneno, óbito

\section{Epidemiological profile of rodenticide-poisoned dogs and cats in a clinic in Belém, Pará}

\begin{abstract}
The primary function of rodenticide is to exterminate the respective animal, which is one of the main disseminators of various diseases in urban environment, which are important for public health, for example leptospirosis. However, most of the time, these substances end up intoxicating pets and can be cause accidentally, when the tutor does not perform the correct management of the rat poison, or intentionally, when It is intended to cause intoxication of the animal, causing various symptomatology in which prognosis is not favorable. Due to the high variety of symptoms, its severity and the high incidence of intoxication cases, an epidemiological survey of casuistry was done in a private clinic in the city of Belém/PA.
\end{abstract}

Keywords: domestic animals, epidemiological research, poison, death

\section{Perfil epidemiológico de perros y gatos envenenados con rodenticidas en una clínica en Belém, Pará}

Resumen. La función principal del rodenticida es exterminar al animal, que es uno de los principales diseminadores de diversas enfermidades em el entorno urbano, importantes para la salud pública, como la leptospirosis. Sin embargo, la mayoría de las veces, estas sustâncias terminan intoxicando a las mascotas y pueden ser causadas accidentalmente cuando el tutor no maneja adecuadaente el veneno para ratas o intencionalmente para 
causar envenenamiento de animales, causando varios sintomas en que el pronóstico no es favorable. Debido a la gran variedad de sintomas, su gravedad y la alta incidencia de casos de intoxicación, se realizó una encuesta epidemiológica de la casuistica em uma clínica privada en la ciudad de Belém, PA.

Palabras clave: animales domésticos, estúdio epidemiológico, veneno, muerte

\section{Introdução}

Os ratos são a principal praga urbana, devido alta taxa de natalidade e adaptabilidade (Magalhães, 2012) e são fontes de doenças (Ferreira et al., 2017). Logo, desenvolveram-se substâncias objetivando controle populacional desses animais dentre eles, os raticidas. Todavia, tal substância atinge animais domésticos. Na maioria dos casos, durante tentativa de eliminação do roedor, a falta de instrução do tutor e ausência de profissional qualificado, causa intoxicação acidental para o animal de companhia. A intoxicação proposital ocorre em cães que faz muito barulho ou em cães de guarda, para facilitar furto (Assis et al., 2009).

Várias substâncias estão nos raticidas, as principais são os cumarínicos e estricnina (Lorenz \& Kornegay, 2006). O Aldicarb é um carbamato de alta toxicidade. A venda é permitida para uso agrícola, mas o mesmo é utilizado como raticida (Araújo et al., 2017). O mecanismo do carbamato consiste na inibição da enzima acetilcolinesterase, gerando acúmulo de acetilcolina nas sinapses, causando hiperestimulação (Araújo et al., 2017). Os sintomas são sialorreia, depressão cardio-pulmonar, coma e morte (Lorenz \& Kornegay, 2006). A estricnina é antagonista da glicina, e possui alta absorção, alcançando medula espinhal, aumentando excitabilidade. O principal sintoma é a convulsão, já a paresia respiratória é responsável pelo óbito (Lorenz \& Kornegay, 2006). Os cumarínicos são anticoagulantes, causam inibição da síntese hepática, dependente da vitamina K1 da protrombina (fator II, VII, IX, X). Efeito antiprotrombina é fulcral para diagnóstico clínico. Pode causar diminuição da agregação plaquetária (Tremori, 2015). O sintoma é hemorragia generalizada, causando choque hipovolêmico e morte (Tremori, 2015).

A vulnerabilidade dos animais, severidade dos sintomas e elevada incidência das intoxicações, objetivou-se realizar o levantamento epidemiológico da casuística de animais intoxicados por raticidas, em uma clínica particular localizada no centro de Belém, Pará.

\section{Materiais e métodos}

Foi realizado um estudo retrospectivo com dados encontrados em uma clínica particular localizada em Belém do Pará, que se localiza no bairro Reduto, região central de Belém. Foram analisados registros do período de 2000 a 2019 e coletados dados das fichas em que o animal foi diagnosticado com intoxicação por rodenticidas. As informações analisadas foram: nome, idade, peso, espécie, bairro, raça, rodenticidas utilizados, óbito e sintomas.

Após a coleta de dados, foi realizada a análise estatística dos mesmos. No caso dos dados de peso e idade, foi necessário dividi-los em classes para tornar viável a análise estatística. Para este fim, foi utilizado o critério de Sturges, dado pela Eq. (01), onde: "K" é um número de classes e "N" é o número de dados (Meyer, 1970).

$$
K=1+3,3 \log _{10}(N)
$$

Para os dados de espécie foi calculada apenas a frequência relativa, visto que as únicas espécies encontradas foram cães e gatos. Da mesma maneira foram calculados os óbitos, não necessitando separálos em classes. Os bairros levados em consideração no gráfico foram aqueles em que houve mais de um caso, sendo que os restantes (em que houve apenas uma ocorrência) ficaram agrupados em "Outros". Para os raticidas encontrados também foi calculada apenas a frequência relativa. Já nos sintomas foi levado em consideração no gráfico apenas os que aparecem em mais de $25 \%$ dos casos, sendo estes: sialorréia, êmese, mioclonia, taquicardia e taquipneia. Além destes, foi adicionado ao gráfico à percentagem de animais que não apresentaram sintomas. 


\section{Resultados e discussões}

Após análise das fichas clínicas dos 37 casos encontrados, foi realizado um levantamento estatístico das seguintes informações do animal: espécie, bairro, se houve óbito, peso, idade, raticida utilizado e sinais clínicos. Com relação à espécie, 91,9\% dos casos foram de caninos contra 8,1\% de felinos; segundo Souza (2003) essa diferença entre os números pode ser explicada pela maior seletividade dos felinos que recusam o alimento quando apresenta odor pouco agradável, além de apresentarem peculiaridades em seu metabolismo, como a deficiência na conjugação de substâncias com o ácido glicurônico na fase dois da biotransformação devido as baixas concentrações de enzimas da família glicoroniltransferase, pois em virtude disso, muitas substâncias que utilizam essa via para o seu metabolismo possuem uma meia-vida prolongada.

Com relação aos bairros, $24,3 \%$ dos tutores residiam no bairro do Reduto, $10,8 \%$ no bairro do Marco, $10,8 \%$ no bairro da Pedreira, $8,1 \%$ no bairro do Umarizal, $8,1 \%$ no bairro do Telégrafo, 5,4\% no bairro da Cidade Velha e 32,4\% em outros bairros. Apesar da maior incidência de roedores, e consequentemente uso de raticidas, ocorrer nas áreas periféricas dos centros urbanos, estes resultados foram influenciados pela localização da clínica veterinária, a qual atende moradores do centro do município de Belém em sua maioria.

Quanto à mortalidade, em apenas 8,1\% dos casos houve óbito, contra 91,9\% de casos em que o animal sobreviveu. Apesar do prognóstico para os quadros de intoxicação por rodenticidas ser ruim, quando diagnosticados precocemente e seguindo adequadamente um protocolo de tratamento, há grandes chances de o animal sobreviver (Talcott \& Dorman, 1997), mesmo em casos mais graves em que haja choque hipovolêmico, desde que os procedimentos terapêuticos estejam prontamente disponíveis (Maroni et al., 2000). Além disso, a letalidade depende, principalmente, da dose, tempo de ingestão e absorção do agente tóxico. Doses excessivas ou ingestão prolongada, associadas á boa absorção pelo trato gastrointestinal comumente levam a quadros mais avançados de hemorragia generalizada e consequente morte (Maroni et al., 2000). O tratamento consiste em estimular o animal a êmese, realização de lavagem gástrica caso a êmese não ocorra, ou administração de carvão ativado por VO. Deve-se também fazer o uso da vitamina K ( $5 \mathrm{mg} / \mathrm{kg}$; SC), após 6-12 horas, administrar em média $1,5 \mathrm{mg} / \mathrm{kg}$ via SC repetindo a cada 12 horas durante 14 dias (Tremori, 2015).

Em sua maioria, os animais mais acometidos apresentavam idade entre 0 e 3 anos $(35,1 \%)$ e pesavam entre 2 e $7 \mathrm{~kg}(40,5 \%)$, seguidos de 13,5\% de animais entre 3 e 6 anos, 13,5\% entre 6 e 9 anos, $10,8 \%$ entre 9 e 12 anos, 2,7\% entre 12 e 15 anos, $8,1 \%$ entre 15 e 18 anos, $16,2 \%$ não foi informada a idade e $27,0 \%$ dos animais apresentavam peso entre 7 e $12 \mathrm{~kg}, 8,1 \%$ entre 12 e $17 \mathrm{~kg}$, $5,4 \%$ entre 22 e $27 \mathrm{~kg}, 2,7 \%$ entre 27 e $32 \mathrm{~kg}$ e 16,2\% não foi informado o peso (Figura 1). A maior incidência dos casos se dá em animais jovens devido ao seu metabolismo imaturo e eliminação deficiente dos agentes tóxicos (Volmer \& Meerdink, 2002), além de serem mais ativos e curiosos assim como os animais de pequeno porte em relação aos de porte maior.

Sobre a etiologia da intoxicação e gênero do raticida, 48,6\% dos casos foram causados por carbamato, conhecido popularmente como chumbinho, 10,8\% por cumarínico, 5,4\% por Brodifacoum e 35,1\% não foi especificado (Figura 2). Segundo estudos realizados por Wang et al. (2007) e Xavier et al. (2007), a principal causa de intoxicações agudas é a ingestão de carbamato, devido a sua fácil aquisição por conta do comércio clandestino dessa substância, seguida dos raticidas cumarínicos.

Os sinais clínicos mais recorrentes foram: sialorréia e mioclonia, presentes em 45,9\% dos casos, êmese $(43,2 \%)$, taquipnéia $(29,7 \%)$, taquicardia $(27,0 \%)$ e 16,2\% não apresentou sintomas (Figura 3 ). Estes dados estão de acordo com estudos de Lorenz \& Kornegay (2006). Dependendo do tipo de raticida envolvido na intoxicação, muitas vezes nenhum sinal clínico é observado em animais sobreviventes (Kerr et al., 1991), ou mesmo a sintomatologia inicial apresentada é muito inespecífica (Berny et al., 2010). 


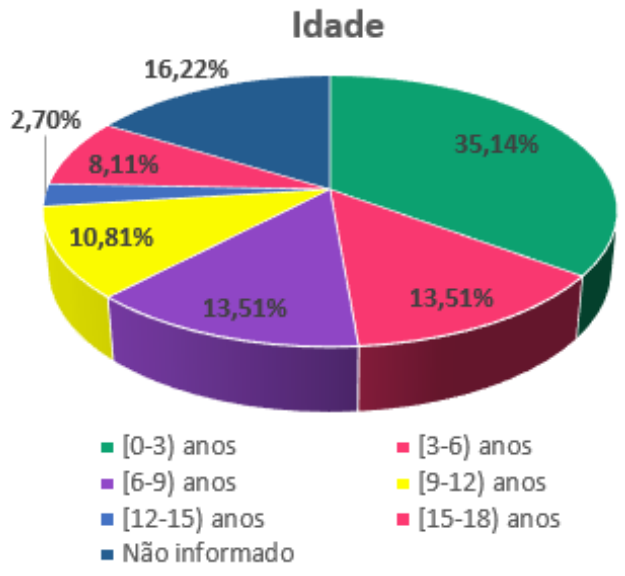

Figura 1. Idade dos animais.

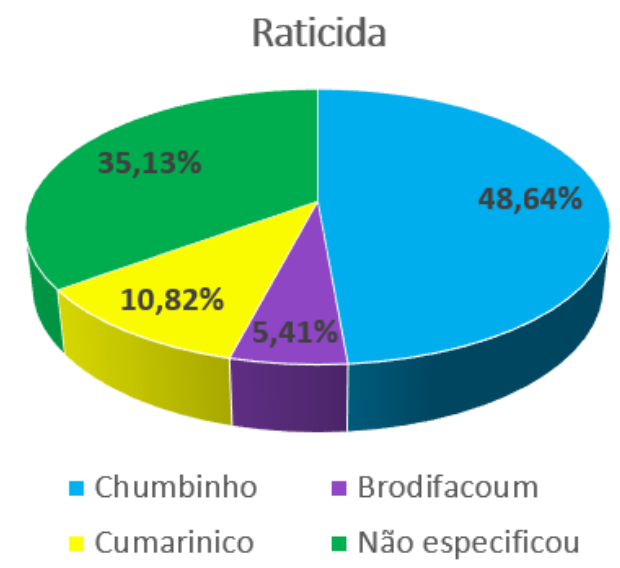

Figura 2. Raticidas encontrados.

\section{Sintomas}

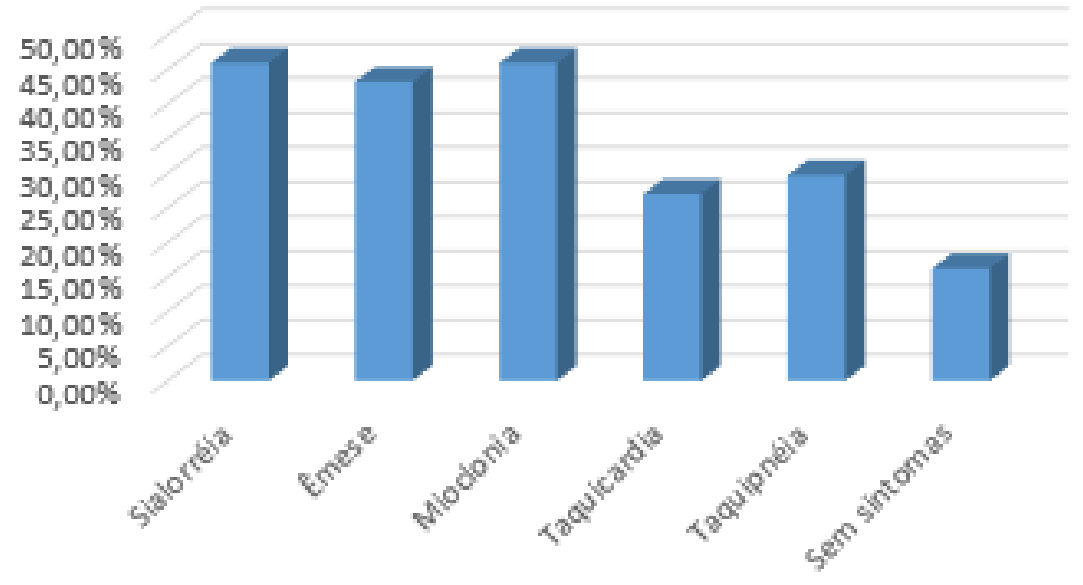

Figura 3. Sintomas relatados

\section{Conclusão}

Diante do exposto, conclui-se que o conhecimento acerca dos principais tipos de rodenticidas utilizados e do levantamento epidemiológico é de suma importância, pois possibilitou ter o conhecimento do tipo de animal mais propenso a ser intoxicado, e dessa forma possuir maior cautela com esse tipo de animal, conscientizando tanto a classe de médicos veterinários quanto os tutores de animais, aumentando dessa forma a cautela acerca do manuseio dos rodenticidas por parte do proprietário, diminuindo dessa forma a intoxicação acidental em animais de companhia

\section{Referências bibliográficas}

Araújo, L. A., Rodrigues, L. M. N., Rodrigues, I. L., Viana, D. A., Rocha, L. L. C. V. \& Santos, L. F. L. (2017). Intoxicação por aldicarb (chumbinho) em um felino - relato de caso. Revista Ciência Animal, 27(2):113-116.

Assis, H. C. S., Hansen, D. T. K. \& Almeida, M. I. M. (2009). Perfil das intoxicações apresentadas por cães e gatos em Curitiba, Paraná. Revista do Conselho Federal de Medicina Veterinária, 15(47):2228.

Berny, P., Caloni, F., Croubels, S., Sachana, M., Vandenbroucke, V., Davanzo, F. \& Guitart, R. (2010). Animal Poisoning in Europe. Part 2: Companion Animals. The Veterinary Journal, 183(3):255-259. 
Ferreira, S. B., Sousa, K. R. S., Castro, V., Lopes, S. T. P., Ferreira, S. B., Feitosa, L. C. S., . . Souza, J. A. T. (2017). Análise soroepidemiológica e fatores de risco associados à Leptospira spp. em bovinos no estado do Piauí. Acta Scientiae Veterinariae, 451-11.

Kerr, L. A., Pringle, J. K., Rohrbach, B. W., Edwards, W. C. \& Offutt, J. E. (1991). Aldicarb toxicosis in a dairy herd. Journal of the American Veterinary Medical Association, 198(9):1636-1639.

Lorenz, M. D. \& Kornegay, J. N. (2006). Neurologia veterinária. Barueri: Manole.

Magalhães, L. E. (2012). A ciência e os animais de laboratório. Revista da Sociedade Brasileira de Ciência em Animais de Laboratório, 1(1):7-13.

Maroni, M., Colosio, C., Ferioli, A. \& Fait, A. (2000). Biological monitoring of pesticide exposure: a review. Introduction. Toxicology, 143(1):1-118.

Meyer, P. L. (1970). Probabilidade: aplicações à estatística: Livros Técnicos e Científicos Editora.

Souza, H. J. M. (2003). Coletâneas em medicina e cirurgia felina. Rio de Janeiro, Brasil: Livros de Veterinária.

Talcott, P. A. \& Dorman, D. C. (1997). Pesticide exposures in companion animals. Veterinary Medicine, 92(2):167-181.

Tremori, T. M. (2015). Cães e gatos: expressão das lesões em intoxicações criminais. Master of Science, Universidade Estadual Paulista, Botucatu, São Paulo, Brasil.

Volmer, P. A. \& Meerdink, G. L. (2002). Diagnostic toxicology for the small animal practitioner. The Veterinary clinics of North America. Small Animal Practice, 32(2):357-365.

Wang, Y., Kruzik, P., Helsberg, A., Helsberg, I. \& Rausch, W.-D. (2007). Pesticide poisoning in domestic animals and livestock in Austria: a 6 years retrospective study. Forensic Science International, 169(2-3):157-160.

Xavier, F. G., Righi, D. A. \& Spinosa, H. S. (2007). Toxicologia do praguicida aldicarb (" chumbinho"): aspectos gerais, clínicos e terapêuticos em cães e gatos. Ciência Rural, 37(4):1206-1211.

Recebido: 4 de dezembro, 2019.

Aprovado: 31 de janeiro, 2019.

Publicado: 10 de março, 2020.

Licenciamento: Este artigo é publicado na modalidade Acesso Aberto sob a licença Creative Commons Atribuição 4.0 (CC-BY 4.0), a qual permite uso irrestrito, distribuição, reprodução em qualquer meio, desde que o autor e a fonte sejam devidamente creditados. 